\title{
Using the Gottwald and Melbourne's 0-1 test and the Hugichi fractal dimension to detect chaos in defective and healthy ball bearings
}

\author{
C. A. Kitio Kwuimy ${ }^{1, *}$, T. Haj Mohamad ${ }^{2, * *}$, and C. Nataraj ${ }^{2, * * *}$ \\ ${ }^{1}$ Department of Engineering Education, College of Engineering and Applied Science, University of Cincinnati, Cincinnati, \\ $\mathrm{OH} 45221$ \\ ${ }^{2}$ Villanova Center for Analytics of Dynamic Systems (VCADS), Villanova University, Villanova, PA 19085, USA
}

\begin{abstract}
The paper considers the identification of chaotic behavior dynamics using the data extracted from an experimental model of rotor supported on rolling elements. A description of the method is provided as well as an illustration using a standard dynamic map. The 0-1 test for chaos and the Higuchi dimension are shown to be effective tool in the identification of chaotic behavior of the system bearing with and without faults.
\end{abstract}

\section{Introduction}

Time series analysis is generally referred to as the transformation of observed dynamic data into insight for making better decisions [1]. The data extracted from the behavior of dynamic systems can be nonperiodic and eventually stochastic. For specific applications these data need to be characterized and analyzed in order to extract information about the system. Several methods have been used in various branches of science including engineering, epidemiology, biology and finance [2-4]. Traditional methods for time series in these domains include statistical analysis and dynamic method which include methods in the time domain, methods in the state space domain and methods in the frequency domain.

The most used frequency analysis method is the Fast Fourier Transform (FFT), which is in fact a linear method based on the analysis of the spectrum of the frequencies in the response of the system. For instance, a periodic system will be completely described by its FFT while for a non-periodic or stochastic system, the broad-banded FFT will give no specific information [4]. State space methods include all methods developed in the state space of the system. These methods involve all state variables of the system dynamics. The key point here is the necessity of the

\footnotetext{
*e-mail: cedrick.kwuimy@uc.edu

**e-mail: thajmoha@villanova.edu

***e-mail: c.nataraj@villanova.edu
}

state space, which in general (when one cannot obtain the measurement of the dynamics variables of the system) needs to be reconstructed from one of the available measurement. State space methods include the Lyapunov exponent, traditional fractal dimensions such as the correlation dimension, and the method of recurrence analysis. Although these methods have been widely used [2-4], the pitfall here is that they require excessive computing in terms of reconstruction of the state space and some of these methods (Lyapunov exponent for example) are very sensitive to noise and required a certain level of advanced expertise from the user $[3,4,7]$. The time domain methods which consist of a simple plot of the data as function of time. Such plot provides preliminaries information about the nature (periodic, stochastic, chaotic) of the data. Additionally, statistical measurement and fractal measurement of the time response have been shown to provide reliable and efficient information about the nonlinear nature of the system.

The later is considered in this paper. We consider a machine fault simulator and collect data for three conditions of the system (healthy and defective with different levels of severity). The objective is to provide additional insight in the system response. In fact, in previous contribution, we used statistical methods and methods of the state space, namely the method of recurrence analysis for similar investigations $[8,9,11-$ 
14]. Extension was made to the detection and classification of faults. This paper is focussed on identification of chaotic response. The 0-1 test for chaos and the Higuchi's fractal dimension are used to analyze the time series. The methods have been previously used in various branches of science, including finance, engineering and biology [7, 10].

The paper is organized as follows. In Section 2, the description of the methods is summarized. In Section 3 , data from healthy and defective experimental systems are analyzed, periodic and chaotic responses are considered. The last section is the conclusion.

\section{Methods of analysis}

\subsection{The 0-1 test for chaos}

The 0 - 1 test for chaos was developed by Gottwald and Melbourne [7] to distinguish between regular and chaotic dynamics in deterministic dynamical systems. Rather than requiring phase space reconstruction which is necessary to apply standard chaotic indicators, the test works directly with the time series and does not involve any preprocessing of the data. The method is based on the estimation of a statistical parameter $K$ which is used to conclude on the periodic $(K \rightarrow 0)$ and chaotic $(K \rightarrow 1)$ nature of the a time signal. To compute $K$ from a time series

$$
X=x(1), x(2), \ldots x(N)
$$

where $N$ is the total number of data collected, the time series is projected into a plane $(p, q)$ defined as

$$
p(n, c)=\sum_{j=1}^{n} \phi(j) \cos j c, q(n, c)=\sum_{j=1}^{n} \phi(j) \sin j c
$$

where $n=1,2, . ., N$ and $c \in(0,2 \pi)$ is an arbitrary constant. The diffusive and non-diffusive behavior of $p(n, c)$ and $q(n, c)$ are used to estimate $K$. For this end, the adjusted mean-square displacement is estimated. It is given by [7]

$$
\begin{aligned}
D(n, c) & =\frac{1}{N} \sum_{j=1}^{N}\left[\Delta_{1}^{2}+\Delta_{2}^{2}\right]-\Phi^{2} \frac{1-\cos n c}{1-\cos c} \\
\Delta_{1} & =p(n+j, c)-p(j, c) \\
\Delta_{2} & =q(n+j, c)-q(j, c) \\
\Phi & =\frac{\lim }{N \rightarrow \infty} \frac{1}{N} \sum_{j=1}^{N} \phi(j)
\end{aligned}
$$

To ensure the limit $N \rightarrow \infty$, in general the limit $n \leq N_{0}$ with $N_{0} \ll N\left(N_{0}=N / 20\right.$ in this paper $)$ is considered. The second term in Eq. (3) is added to minimize eventual fluctuation in $M(n, c)$. For each values of $c$, the parameter $K_{c}$ is estimated as

$$
K_{c}=\frac{\operatorname{cov}(\zeta, \delta)}{\sqrt{\operatorname{var}(\zeta) \operatorname{var}(\delta)}}
$$

where $\zeta=1,2, . ., N_{0}$ and $\delta=$ $D(c, 1), D(c, 2), . . D\left(c, N_{0}\right)$. The final value of $K$ is estimated as the median of all $K_{c}=f(c)$. The use of the median instead of the average value is justified by the fact that, it avoid large drift caused by possible resonance in a specific value of $c$. See Refs. [7] for illustrations.

\subsection{Higuchi fractal dimension}

The Higuchi's fractal dimension is a powerful fractal measurement of the structure of data [10]. The specificity here is that, unless other fractal dimensions which are estimated in the (reconstructed) state space, the Huguichi's fractal dimension can be computed from a single time series. The Higuchi's fractal dimension has been used as dynamic tool in several applications, including mechanical engineering and geophysics [10].

For the time series in Eq. (1), one can extract different short time series as

$$
X_{k}^{m}=x(m), x(m+k), \ldots x\left(m+\left\lceil\frac{n-m}{k}\right\rceil k\right)
$$

where $\lceil$.$\rceil is the ceiling function. The length of the$ curve associated to each new time series $X_{k}^{m}$ is then computed as the sum of algebraic distances between consecutive elements of the new time series, that is

$$
L_{m}(k)=\sum_{i=1}^{\left\lceil\frac{n-m}{k}\right\rceil}(x(m+i k)-x(m-(i-1) k))
$$

The dimension is computed using the following scaling relation

$$
<L(k)>\propto k^{-D}
$$

where $\langle L(k)>$ is the average lengths of the segment used to measure the total length of the time series at scale $k$, and $L(k)$ is the normalized length defined as

$L_{m}=\frac{N-1}{\left\lceil\frac{n-m}{k}\right\rceil k} \sum_{i=1}^{\left\lceil\frac{n-m}{k}\right\rceil}(x(m+i k)-x(m-(i-1) k))$

The Higuchi fractal dimension is then estimated as the slope of the least square linear fit of $\langle\operatorname{Ln}(L(k))\rangle=$ $\operatorname{Ln}(1 / k)^{\cdot}$ 


\section{Detection of chaotic behavior in bearings}

\subsection{The experimental setup and data collection}

The machine fault simulator used as experimental setup is shown in Fig. 1. It consists of a motor with a closed loop speed control connected to a shaft supported on rolling element bearings. The system can be loaded using a mass and balancing disks. A load mass of $5 \mathrm{~kg}$ is used in this research. The balancing disk has two rows of screw holes drilled near the edge into which different masses can be inserted to balance the shaft. Data are captured with three different bearings. Two of these bearings have an outer race defect with different fault size and one bearing does not have any defect. The signals from the experimental setup are collected with accelerometers placed at the bearing case.

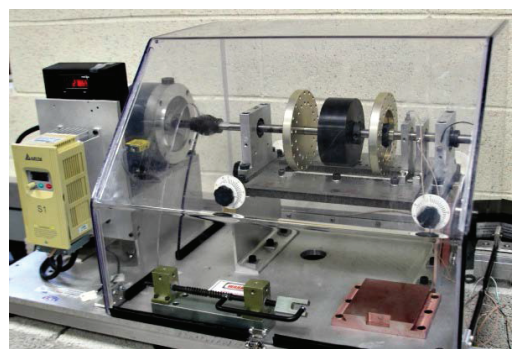

Figure 1. Experimental setup

\subsection{Data analysis and evidence of chaos}

In this section, data are collected at speed $40 \mathrm{~Hz}$ for the healthy and defective conditions. Bearings with outer race defects have impulses excited by the rolling element entering the defect. Because of these impulses there is a significant difference between the signals from bearings with and without defects.

The $(p(n, c), q(n, c))$ projection of the data are shown in Fig. 2 for the system with small defect. The diffusive structure of the plot is clearly a signature of a non-deterministic dynamics. For different values of $c$, the global behavior of Fig. 3 is obtained and for this specific set of parameters, the corresponding median is quite identical $(K=0.99)$ for the three states of the system as shown in the figures.

The graphs of Fig. 3 are obtained using the procedure for described above for the Higuchi dimension. The slopes are obtained respectively as $H=1.96$ for the healthy system, $H=1.81$ and $H=1.95$ for the

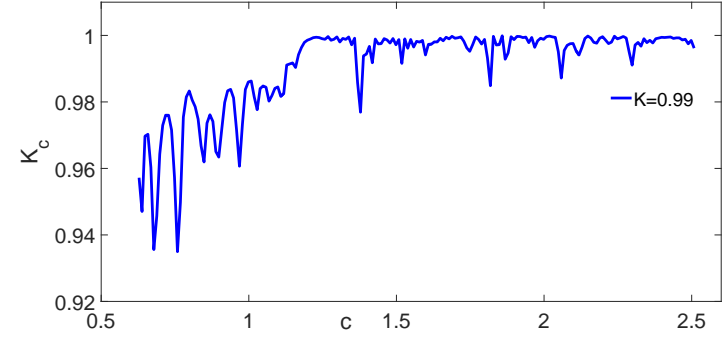

Figure 2. Estimation of the $0-1$ test parameter of the bearing system with small defect

system with small and large defects. The figure also shows an extension of the procedure for different set of data corresponding to rotor speed $50 \mathrm{~Hz}$ and $10 \mathrm{~Hz}$. The results are structurally characteristic of a nonperiodic response.

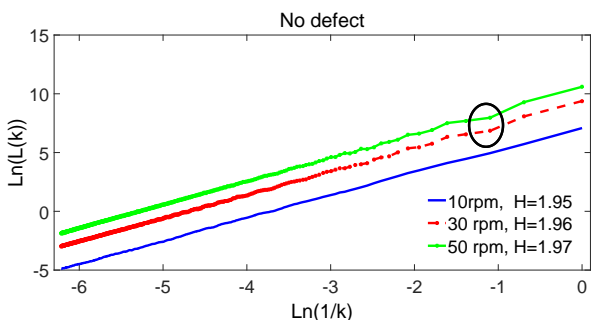

(a)

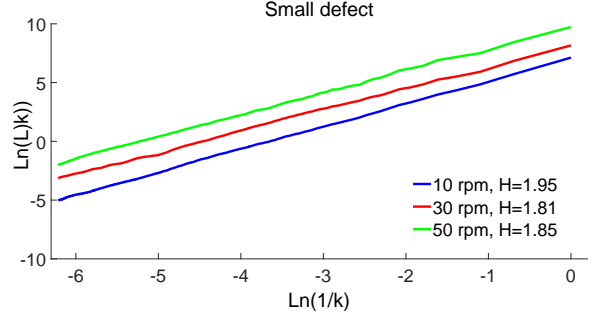

(b)

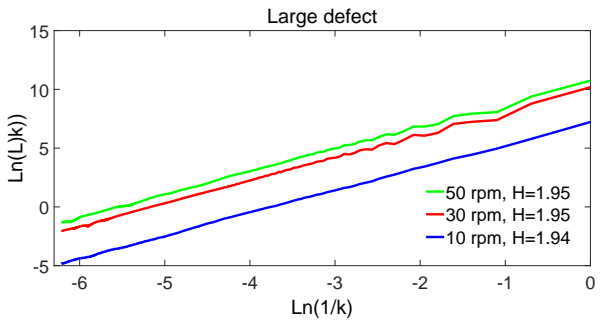

(c)

Figure 3. Estimation of the Higuchi fractal dimension. (a): Healthy system. (b): System with small defect. (c): System with large defect.

As in previous contributions [8], the structure of the time response of the bearing can be quite complex. This complexity was initially captured through the structure of the recurrence plots matrix and quantify 
using the so-called recurrence quantification parameters. The results obtained in this paper, provide a more "simplistic" identification of chaos. The Higuchi dimension can be seen here as a graphical tool (from which a quantification can be deduced) and it is complement with the $0-1$ test.

\section{Conclusion}

The main objective of this work was to identify chaotic response of bearing system with and without defects. Two method were used for this main. The 0-1 test for chaos the Higuchi fractal dimension. The data were analyzed and the methods have been shown to be an effective tool for the identification of chaotic behavior.

The results obtained in this paper are extensions of results obtained in other applications, where the 0-1 test and the Higuchi dimension were used either separably to analyze experimental data. One key relevance of this work is the relative simplicity of the methods and their robustness. Thus, they appear as strong candidate for the characterization of experimental data as well as fault analysis. In fact, in these two applications, simpler and robust methods are always advantageous (computational cost and robustness). Current investigations are carried out to analyze the effects of noise and the dynamics of the system under various parameter configurations.

\section{Acknowledgments}

Part of this work was done during the Postdoctoral fellow of C.A.K. Kwuimy at the VCADS. He would like to thank Dr. C. Nataraj and the US Office of Naval Research for the financial support (grant N00014-13-1-0485)

\section{References}

[1] E.A. Boyd, Analytics 7, 6 (2012)

[2] A. Galka, Topics in Nonlinear Time Series Analysis (World Scienti, 2000)

[3] H. Kantz, T. Schreiber, Nonlinear Time Series Analysis (University Press-Cambridge, 2004)
[4] H.D.I. Abarbanel, Analysis of Observed Chaotic Data (Springer-Verlag, New York, 1996)

[5] C.A.K. Kwuimy, C. Nataraj, Structural Nonlinear Dynamics \& Control and Diagnosis (Springer-Verlag, 2015), pp. 97-123

[6] N. Marwan, M.C. Romano, M. Thiel, J. Kurths, Physics Reports 438(5-6), 237 (2007)

[7] A.G. Gottwald, I. Melbourne, SIAM Journal of Applied Dynamical Systems 8, 129 (2009)

[8] C.A.K. Kwuimy, M. Samadani, K. Kappaganthu, C. Nataraj, in International Conference on $\mathrm{Vi}$ bration Engineering and Technology of Machinery (2014)

[9] T. Haj Mohamad, M. Samadani, C. Nataraj, Journal of Vibration and Acoustics, 140(6), 061009 (2018).

[10] T. Higuchi, Physica, 23, 277 (1988)

[11] M. Samadani, T. Haj Mohamad, C. Nataraj, Feature Extraction for Bearing Diagnostics Based on the Characterization of Orbit Plots With Orthogonal Functions, in ASME 2016 International Design Engineering Technical Conferences and Computers and Information in Engineering Conference, pp. V008T10A026V008T10A026

[12] T. Haj Mohamad, C.A.K. Kwuimy, C. Nataraj, Discrimination of Multiple Faults in Bearings Using Density-Based Orthogonal Functions of the Time Response, in ASME 2017 International Design Engineering Technical Conferences and Computers and Information in Engineering Conference, pp. V008T12A035-V008T12A035

[13] T.H. Mohamad, Y. Chen, Z. Chaudhry, C. Nataraj, Journal of Software Engineering and Applications 11, 181 (2018)

[14] T. Haj Mohamad, A.A. Cavalini, V. Steffen, C. Nataraj, Detection of Cracks in a Rotating Shaft Using Density Characterization of Orbit Plots, in Proceedings of the 10th International Conference on Rotor Dynamics - IFToMM, edited by K.L. Cavalca, H.I. Weber (Springer International Publishing, Cham, 2019), pp. 90-104, ISBN 978-3-319-99268-6 